\title{
ON THE TENSOR PRODUCT OF QUATERNION ALGEBRAS OF CHARACTERISTIC TWO
}

\author{
by P. MAMMONE
}

(Received 13 May, 1986)

1. Introduction. The purpose of this note is to generalize to fields of characteristic two the results obtained in [4]. We obtain necessary and sufficient conditions involving quadratic forms for certain tensor products of quaternion algebras to be division algebras.

We apply this to show, as in [4], that the Albert criterion does not generalize to tensor products of more than two quaternion algebras.

More precisely, let $k$ be a field of characteristic two, $a \in k$ and $b \in k^{\times}(=k-\{0\})$; we denote by $[a, b)_{k}$ the quaternion $k$-algebra generated by two elements $e_{1}$ and $e_{2}$ subject to the relations:

$$
\begin{aligned}
\mathscr{P}\left(e_{1}\right): & =e_{1}^{2}+e_{1}=a, \\
e_{2}^{2} & =b, \\
e_{2} e_{1} & =e_{1} e_{2}+e_{2} .
\end{aligned}
$$

Let us also denote by $[a, b]$ the quadratic form $a X^{2}+X Y+b Y^{2}$. To the tensor product of quaternion algebras

$$
T=\left[a_{1}, b_{1}\right)_{k} \otimes \ldots \otimes\left[a_{n}, b_{n}\right)_{k},
$$

we associate the quadratic form

$$
Q_{T}=\left[1, a_{1}+\ldots+a_{n}\right] \underset{i=1}{\stackrel{n}{\perp}}\left\langle b_{i}\right\rangle\left[1, a_{i}\right] .
$$

In fact, for $n=1$ and 2, it is well known that $T$ has zero divisors if and only if $Q_{T}$ is isotropic, see [1, p. 29 and p. 131]. In $\S 3$, we show that this assertion is false for $n \geqslant 3$. A similar question was first proposed by $\mathrm{D}$. W. Lewis over fields of characteristic different from two, see [3] and [4]. Note that $Q_{T}$ is, as in [3] and [4], the (alternating) sum of the reduced norms of the quaternion algebras $\left[a_{i}, b_{i}\right)$ minus the $(n-1)$ obvious hyperbolic planes.

2. Generic extensions of division algebras. Let $X_{1}, \ldots, X_{n-1}, Y_{1}, \ldots, Y_{n-1}, Z$ be independent indeterminates over $k$ (with $n \geqslant 3$ ) and let also

$$
F=k\left(X_{1}, \ldots, X_{n-1}, Y_{1}, \ldots, Y_{n-1}, Z\right) .
$$

For $f \in k\left(X_{1}, \ldots, X_{n-1}\right)$ and $g \in k\left(X_{1}, \ldots, X_{n-1}, Z\right)$, we define

and

$$
T_{f}:=\left[X_{1}, Y_{1}\right)_{F} \otimes \ldots \otimes\left[X_{n-1}, Y_{n-1}\right)_{F} \otimes[f, Z)_{F}
$$

$$
T_{g}^{\prime}:=\left[X_{1}, Y_{1}\right)_{F} \otimes \ldots \otimes\left[X_{n-1}, Y_{n-1}\right)_{F} \otimes[Z, g)_{F} .
$$

Glasgow Math. J. 30 (1988) 111-113. 
TheOREM A. (i) $T_{f}$ is a division algebra if and only if

$$
f \notin \mathscr{P}\left(k\left(\mathscr{P}^{-1}\left(X_{1}\right), \ldots, \mathscr{P}^{-1}\left(X_{n-1}\right)\right)\right) .
$$

(ii) $T_{g}^{\prime}$ is a division algebra if and only if $g$ is not represented by the quadratic form $[1, Z]$ over $k\left(X_{1}, \ldots, X_{n-1}, Z\right)$.

THEOREM B. (i) $Q_{T}$ is anisotropic over $F$ if and only if the quadratic form $\left[1, X_{1}+\ldots+X_{n-1}+f\right]$ is anisotropic over $k\left(X_{1}, \ldots, X_{n-1}\right)$ and $f \notin \mathscr{P}\left(k\left(X_{1}, \ldots, X_{n-1}\right)\right)$.

(ii) $Q_{T_{s}^{\prime}}$ is anisotropic over $F$ if and only if the quadratic form

$$
\left[1, X_{1}+\ldots+X_{n-1}+Z\right] \perp\langle g\rangle[1, Z]
$$

is anisotropic over $k\left(X_{1}, \ldots, X_{n-1}, Z\right)$.

The proofs will follow by repeated use of the following results.

Lemma A. Let $A$ be a division algebra over $k, c \in k$ and $X$ an indeterminate over $k$ : then we have:

(i) $A \otimes_{k}[c, X)_{k(x)}$ is a division algebra if and only if $A \otimes_{k} k\left(\mathscr{P}^{-1}(c)\right)$ is a division algebra;

(ii) $A \otimes_{k}[X, c)_{k(x)}$ is a division algebra if and only if $A \otimes_{k} k(\sqrt{ } c)$ is a division algebra.

Proof. (i) If $A \otimes_{k} k\left(\mathscr{P}^{-1}(c)\right)$ is not a division algebra, we easily see that

$$
\mathscr{P}^{-1}(c) \otimes 1+1 \otimes \mathscr{P}^{-1}(c)
$$

is a zero divisor of $A \otimes_{k}[c, X)_{k(x)}$. Now suppose that $D:=A \otimes_{k} k$ $\left(\mathscr{P}^{-1}(c)\right)$ is a division algebra. We first observe that the quaternion algebra $[c, X)_{k(x)}$ can be written in the form $k\left(\mathscr{P}^{-1}(c)\right)(X ; \sigma)$, where $\sigma$ is the non-trivial $k$-automorphism of $k\left(\mathscr{P}^{-1}(c)\right)$.

Since $D$ is a division algebra, we can extend $\sigma$ to $D$ in such a way that $\sigma_{\mid A}=1_{A}$. But then we remark that $A \otimes_{k}[c, X)_{k(x)}$ is nothing else than $D(X ; \sigma)$.

(ii) If $A \otimes_{k} k\left(V_{c}\right)$ is not a division algebra, we easily see that $V_{c} \otimes 1+1 \otimes V_{c}$ is a zero divisor of $A \otimes_{k}[X, c)_{k(x)}$. Suppose now that $D^{\prime}:=A \otimes_{k} k(\sqrt{ } c)$ is a division algebra. Let $e$ be the basis element of $[X, c)_{k(x)}$ such that $e^{2}+e=x$ then, if we put $t=c^{-1} V_{c} e$, we can verify the following relations: $t^{2}=c^{-1} X \in k(x)$ and $t \vee c=\sqrt{ } c t+1$. This shows that we can write the quaternion algebra $[X, c)_{k(x)}$ in the form $k(\sqrt{ } c)(t ; \delta)$, where $\delta$ is the derivation defined by $\delta(\sqrt{ } c)=1$. Since $D^{\prime}$ is a division algebra, we can extend $\delta$ to $D^{\prime}$ so that $\delta_{\mid A}=0$. But then, as in the previous case, $A \bigotimes_{k}[X, c)_{k(x)}$ is nothing else than $D(t ; \delta)$.

Lemma B. Let $Q_{1}$ and $Q_{2}$ be two quadratic forms over $k$, and $X$ an indeterminate over $k$. Then $Q_{1} \perp\langle x\rangle Q_{2}$ is anisotropic over $k(x)$ if and only if $Q_{1}$ and $Q_{2}$ are anisotropic over $k$.

Proof. We take first a representation of $Q_{1}$ and $Q_{2}$ with respect to the symplectic 
basis and then proceed, as for the case of characteristic different from two (see [2, $\mathrm{p}$. 273]), by a degree argument. Details are left to the reader.

Proof of Theorem A. (i) By induction and Lemma A(i), we see that $T_{f}$ is a division algebra if and only if the quaternion algebra $[f, Z)$ is a division algebra over $k\left(\mathscr{P}^{-1}\left(X_{1}\right), \ldots, \mathscr{P}^{-1}\left(X_{n-1}\right), Z\right)$. But this condition is equivalent to the following (see the introduction): $[1, f] \perp\langle Z\rangle[1, f]$ is an anisotropic quadratic form over $k\left(\mathscr{P}^{-1}\left(X_{1}\right), \ldots, \mathscr{P}^{-1}\left(X_{n-1}\right), Z\right)$. Applying now Lemma B for $X=Z$, we see that this condition holds if and only if $[1, f]$ is an anisotropic quadratic form over $k\left(\mathscr{P}^{-1}\left(X_{1}\right), \ldots, \mathscr{P}^{-1}\left(X_{n-1}\right)\right)$, i.e. $f \notin \mathscr{P}\left(k\left(\mathscr{P}^{-1}\left(X_{1}\right), \ldots, \mathscr{P}^{-1}\left(X_{n-1}\right)\right)\right)$.

(ii) By induction and Lemma $A\left(\right.$ ii), we see that $T_{g}^{\prime}$ is a division algebra if and only if the quaternion algebra $[Z, g)$ is a division algebra over $k\left(X_{1}, \ldots, X_{n-1}, Z, \sqrt{Y_{1}}, \ldots, \sqrt{Y_{n-1}}\right)$. This last condition is clearly equivalent to the following: $g$ is not represented by the quadratic form $[1, Z]$ over

$$
k\left(X_{1}, \ldots, X_{n-1}, Z, \sqrt{Y_{1}}, \ldots, \sqrt{Y_{n-1}}\right),
$$

and so, if and only if $g$ is not represented by $[1, Z]$ over $k\left(X_{1}, \ldots, X_{n-1}, Z\right)$.

Proof of Theorem B. Use induction and Lemma B.

REMARK. The quadratic form $\left[1, X_{1}+\ldots+X_{n-1}+f\right]$ is isotropic over $k\left(X_{1}, \ldots, X_{n-1}\right)$ if and only if $X_{1}+\ldots+X_{n-1}+f \in \mathscr{P}\left(k\left(X_{1}, \ldots, X_{n-1}\right)\right)$. Since $X_{1}+\ldots+X_{n-1} \in \mathscr{P}\left(k\left(\mathscr{P}^{-1}\left(X_{1}\right), \ldots, \mathscr{P}^{-1}\left(X_{n-1}\right)\right)\right.$, this last condition implies that $f \in \mathscr{P}\left(k\left(\mathscr{P}^{-1}\left(X_{1}\right), \ldots, \mathscr{P}^{-1}\left(X_{n-1}\right)\right)\right.$. This shows that if $T_{f}$ is a division algebra then $Q_{T_{f}}$ is anisotropic.

3. The counterexamples. In the introduction we said that the equivalence, $T$ is a division algebra if and only if $Q_{T}$ is anisotropic, holds if $T$ is a quaternion algebra or a tensor product of two quaternion algebras. We now provide counterexamples to both implications for $n \geqslant 3$. Applying Theorems $\mathrm{A}$ and $\mathrm{B}$, we can see that

(1) for $f=X_{1}+\ldots+X_{n-1}, T_{f}$ is not a division algebra and $Q_{T_{f}}$ is anisotropic;

(2) for $g=X_{1}+\ldots+X_{n}+Z$, $T_{g}^{\prime}$ is a division algebra and $Q_{T_{g}^{\prime}}$ is isotropic.

\section{REFERENCES}

1. R. Baeza, Quadratic forms over semilocal rings, Lecture Notes in Mathematics. 655 (Springer, 1978).

2. T. Y. Lam, The algebraic theory of quadratic forms, (Benjamin, 1973).

3. D. W. Lewis, A note on Clifford algebras and central division algebras with involution, Glasgow Math. J. 26 (1985), 171-176.

4. P. Mammone and J. P. Tignol, Clifford division algebras and anisotropic quadratic forms: two counterexamples, Glasgow Math. J. 28 (1986), 227-228.

Université de Mons-Hainaut

B-7000 Mons 